Analysis of power output for piezoelectric energy harvesting systems

This article has been downloaded from IOPscience. Please scroll down to see the full text article.

2006 Smart Mater. Struct. 151499

(http://iopscience.iop.org/0964-1726/15/6/001)

The Table of Contents and more related content is available

Download details:

IP Address: 140.112.113.225

The article was downloaded on 22/12/2008 at 01:20

Please note that terms and conditions apply. 


\title{
Analysis of power output for piezoelectric energy harvesting systems
}

\author{
Y C Shu ${ }^{1}$ and I C Lien \\ Institute of Applied Mechanics, National Taiwan University, Taipei 106, Taiwan, \\ Republic of China \\ E-mail: yichung@ @pring.iam.ntu.edu.tw
}

Received 1 April 2006, in final form 11 August 2006

Published 25 September 2006

Online at stacks.iop.org/SMS/15/1499

\begin{abstract}
Power harvesting refers to the practice of acquiring energy from the environment which would be otherwise wasted and converting it into usable electric energy. Much work has been done on studying the optimal AC power output, while little has considered the AC-DC output. This article investigates the optimal AC-DC power generation for a rectified piezoelectric device. In contrast with estimates based on various degrees of approximation in the recent literature, an analytic expression for the AC-DC power output is derived under steady-state operation. It shows that the harvested power depends on the input vibration characteristics (frequency and acceleration), the mass of the generator, the electrical load, the natural frequency, the mechanical damping ratio and the electromechanical coupling coefficient of the system. An effective power normalization scheme is provided to compare the relative performance and efficiency of devices. The theoretical predictions are validated and found to be in good agreement with both experimental observations and numerical simulations. Finally, several design guidelines are suggested for devices with large coupling coefficient and quality factor.
\end{abstract}

\section{Introduction}

The development of wireless sensor and communication node networks has received a great deal of interest in research communities over the past few years. Applications envisioned from these node networks include building structural health monitoring and environmental control systems, smart homes and tracking devices on animals in the wild [23, 32]. However, as the networks increase in number and the devices decrease in size, the proliferation of these autonomous microsensors raises the problem of an effective power supply. The conventional solution is to use electrochemical batteries for power. However, batteries can not only increase the size and weight of microsensors but also suffer from the limitations of a brief service life and the need for constant replacement, which is not acceptable or even possible for many practical applications.

On the other hand, simultaneous advances in lowpower electronic design and fabrication have reduced power requirements for individual nodes. It has been predicted that

\footnotetext{
1 Author to whom any correspondence should be addressed.
}

power consumption could be reduced to tens to hundreds of microwatts depending on the application [3]. This opens the possibility for self-powered sensor nodes, and the need to power remote systems or embedded devices independently has motivated many research efforts focused on harvesting electrical energy from various ambient sources. These include solar power, thermal gradients and vibration [37]. Among these energy scavenging sources, mechanical vibration is a potential power source that is abundant enough to be of use, is easily accessible through microelectromechanical systems (MEMS) technology for conversion to electrical energy, and is ubiquitous in applications from small household appliances to large infrastructures $[36,41]$.

Vibration energy can be converted into electrical energy through piezoelectric, electromagnetic and capacitive transducers. Among them, piezoelectric vibration-toelectricity converters have received much attention, as they have high electromechanical coupling and no external voltage source requirement, and they are particularly attractive for use in MEMS [13, 30, 39]. As a result, the use of piezoelectric materials for scavenging energy from ambient vibration sources has recently seen a dramatic rise for power 
harvesting. For example, early work at the MIT Media Lab investigated the feasibility of harnessing energy parasitically from various human activities [45]. It was later confirmed that energy generated by walking can be collected using piezoelectric ceramics [40]. Since then, piezoelectric elements used for power harvesting in various forms of structure have been proposed to serve specific purposes. Elvin et al $[6,7]$ and $\mathrm{Ng}$ and Liao [27] have used the piezoelectric element simultaneously as a power generator and a sensor. They have evaluated the performance of the piezoelectric sensor to power wireless transmission and validated the feasibility of the self-powered sensor system. Roundy and Wright [38] have analysed and developed a piezoelectric generator based on a two-layer bending element and used it as a basis for generator design optimization. Similar works based on cantilever-based devices using piezoelectric materials to scavenge vibration energy include [4, 25, 26, 51, 53].

Instead of 1-D design, Kim et al [16, 17] and Ericka et al [8] have modeled and designed piezoelectric plates (membranes) to harvest energy from pulsing pressure sources. Other harvesting schemes include the use of long strips of piezoelectric polymers (energy harvesting eel) in ocean or river-water flows $[1,46]$, the use of piezoelectric 'cymbal' transducers operated in the $\{3-3\}$ mode $[14,15]$ and the use of a piezoelectric windmill for generating electric power from wind energy [31]. Jeon et al [13] have successfully developed the first PZT MEMS power-generating device. Related works on modeling and design considerations for MEMS-scale piezoelectric-based energy harvesters can be found in $[5,24,33]$.

Most published results have reported measurements of output voltage or power, while few have quantified the efficiency of their devices. Umeda et al [48, 49] and Goldfarb and Jones [9] have studied the efficiency of electric power generation with piezoelectric elements operated in the $\{3-$ $1\}$ and \{3-3\} modes, respectively. Recently, Richards et al [34] have provided an analytic formula to predict power conversion efficiency, and showed that it depends on the electromechanical coupling coefficient and quality factor of the device. Roundy [35] has further provided a general theory of the effectiveness of vibration-based energy harvesting which can be applied to electromagnetic, piezoelectric, magnetostrictive and electrostatic transducer technologies. In addition, when a power harvester is applied to a system, it gives rise to an additional damping effect. Lesieutre et al [22] have pointed out that the damping added to a vibrating structure is due to the removal of electrical energy from the system. They have shown that the power harvesting system works similarly to a shunt damping system, except that the energy is stored instead of dissipated [20, 44].

The research works cited above focus mainly on developing optimal energy harvesting structures. However, the electrical outputs of these devices in many cases are too small to power electrical devices directly. Thus, the methods of accumulating and storing parasitic energy are also the key to developing self-powered systems. Sodano et al [42, 43] have investigated several piezoelectric power harvesting devices and the methods of accumulating energy by utilizing either a capacitor or a rechargeable battery. Ottman et al [28, 29] have developed highly efficient electrical circuits to store the generated charge or present it to the load circuit. They have claimed that at high levels of excitation the power output can be increased by as much as $400 \%$. In contrast to the linear load impedance adaptation by [28, 29], Guyomar et al [10] and Lefeuvre et al $[18,19]$ have developed a new power flow optimization principle based on the extraction of the electric charge produced by a piezoelectric element, synchronized with the mechanical vibration operated at the steady state. They have claimed that the harvested electrical power may be increased by as much as $900 \%$ over the standard technique. Badel et al [2] have extended their work to the case of pulsed excitation.

In this paper, we propose an analysis of AC-DC power output for a rectified piezoelectric harvester. Many published results studying the conversion of energy from the oscillating mass to electricity have adopted a simple model proposed by Williams and Yates [5, 13, 36, 39, 52]. It is based on the assumption that the electrical damping term is linear and proportional to the velocity; however, this hypothesis may not be strictly valid in many cases. In addition, much work has been done on studying the optimal AC power flow, while little has considered the AC-DC power output. The former includes $[5,24,25,30,34,38,44]$, while the latter has been studied recently in $[10,19,28]$. As the electronic load requires a stabilized DC voltage while a vibrating piezoelectric element generates an AC voltage, the desired output needs to be rectified, filtered and regulated to ensure electrical compatibility. Thus, it is of importance to investigate the optimal AC-DC power output to reflect the real electrical performance in many practical applications.

Specifically, we study the steady-state response of a piezoelectric generator connected to an AC-DC rectifier followed by a filtering capacitance and a resistor. This problem has recently been studied by Ottman et al [28] and Guyomar et al [10]. The former assumed that the vibration amplitude is not affected by the load resistance while the latter hypothesized that the periodic external excitation and the speed of mass are in phase. In contrast with estimates based on these two approaches, we take into account the global behaviour of the electromechanical system and derive a completely new analytic expression of AC-DC power output in section 2. We show that the harvested power depends explicitly on a number of non-dimensionless parameters. With it, an effective power normalization scheme is provided and can be used to compare power harvesting devices of various sizes and with different vibration inputs to estimate efficiencies. In section 3, we derive the criterion for optimal load and power and study the asymptotic behaviour of power output for devices operated at the short and open circuit resonances, respectively. We show that selection of the correct operation frequency is important for achieving the maximal power flow, while this effect has been neglected in many other approaches. We next validate our theoretical predictions by both experimental results and numerical simulations and find good agreement in section 4 . In addition, we find that the discrepancies among these approaches become significant when the coupling coefficient and quality factor of the system are large. Finally, several design guidelines are recommended from our predictions. We conclude in section 5 with a discussion. 


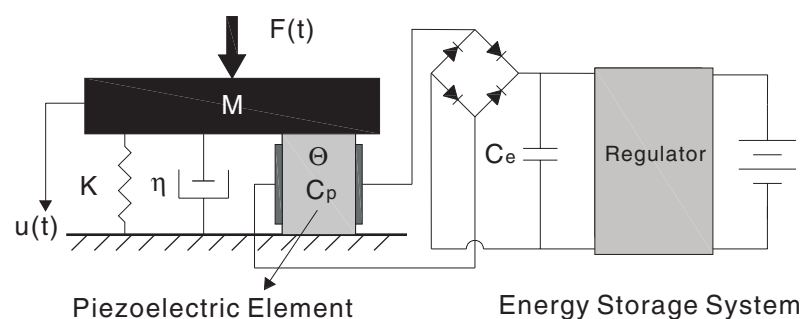

Figure 1. An equivalent model for a piezoelectric vibration energy harvesting system.

\section{Harvesting model}

\subsection{Governing equations}

A piezoelectric energy harvester is often modeled as a mass + spring + damper + piezo structure together with an energy storage system schematically shown in figure 1 $[19,28,34]$. It consists of a piezoelectric element coupled to a mechanical structure and is connected to a storage circuit system. In this approach, an effective mass $M$ subjected to an applied forcing function $F(t)$ is bounded on a spring of effective stiffness $K$, on a damper of coefficient $\eta$, and on a piezoelectric element characterized by effective piezoelectric coefficient $\Theta$ and capacitance $C_{p}$. For example, consider a triple-layer bender mounted as a cantilever beam with polarization poled along the thickness direction as shown in figure 2. The electric field is generated through the direction of thickness of the piezoelectric layers while strain is in the axial direction; consequently, the transverse, or $\{3-1\}$, mode is utilized. The effective coefficients related to material constants and structural geometry can be derived using the modal analysis $[11,50]$

$M=\beta_{M}\left(m_{p}+m_{b}\right)+m_{a}$,

$K=\beta_{K} S\left\{\left(\frac{2}{3} \frac{t^{3}}{L^{3}}+\frac{h t^{2}}{L^{3}}+\frac{1}{2} \frac{t h^{2}}{L^{3}}\right) C_{p_{11}}^{E}+\frac{1}{12} \frac{h^{3}}{L^{3}} C_{b_{11}}^{E}\right\}$,

$\Theta=\beta_{\Theta} \frac{S(h+t)}{2 L} e_{31}$,

$C_{p}=\frac{S L}{2 t} \varepsilon_{33}^{S}$,

where $\beta_{M}, \beta_{K}$ and $\beta_{\Theta}$ are constants derived from the Rayleigh-Ritz approximation, $e_{31}$ and $\varepsilon_{33}^{S}$ are the piezoelectric and clamped dielectric constants, $S$ and $L$ are the width and axial length of the cantilever beam, $t$ and $h, C_{p_{11}}^{E}$ and $C_{b_{11}}^{E}, m_{p}$ and $m_{b}$ are the thicknesses, elastic moduli and masses of the piezoelectric and central passive layers, and $m_{a}$ is the attached mass. We have performed a series of experiments on a PZT triple-layer bender with configuration similar to figure 2 to validate our prediction in section 4.2. Another less common piezoelectric power generator operated in the longitudinal or $\{3-3\}$ mode has been developed recently by [13]. The advantage of utilizing this mode is that the longitudinal piezoelectric effect is usually much larger than the transverse effect $\left(d_{33}>d_{31}\right)$.

A vibrating piezoelectric element generates an AC voltage while the electrochemical battery needs a stabilized DC voltage. This requires an energy harvesting circuit to ensure electrical compatibility. In figure 1, an AC-DC rectifier followed by a filtering capacitance $C_{e}$ is added to smooth the DC voltage. A controller placed between the rectifier output and the battery is included to regulate the output voltage. A simplified energy harvesting circuit shown in figure 3 is commonly chosen for design analysis. Note that the regulation circuit and battery are replaced with an equivalent resistor $R$ and $V_{c}$ is the rectified voltage across it. The rectifying bridge is assumed to be perfect in the following study.

Let $u$ be the displacement of the mass $M$ and $V_{p}$ the voltage across the piezoelectric element. The governing equations of the vibrator can be obtained by the conventional modal analysis $[5,44]$ :

$$
\begin{gathered}
M \ddot{u}(t)+\eta \dot{u}(t)+K u(t)+\Theta V_{p}(t)=F(t), \\
-\Theta \dot{u}(t)+C_{p} \dot{V}_{p}(t)=-I(t) .
\end{gathered}
$$

An AC-DC harvesting circuit is connected to the power generator, as shown in figure $3, I(t)$ is the current flowing into this circuit and is related to the rectified voltage $V_{c}$ by

$I(t)= \begin{cases}C_{e} \dot{V}_{c}(t)+\frac{V_{c}}{R} & \text { if } V_{p}=V_{c}, \\ -C_{e} \dot{V}_{c}(t)-\frac{V_{c}}{R} & \text { if } V_{p}=-V_{c}, \\ 0 & \text { if }\left|V_{p}\right|<V_{c} .\end{cases}$

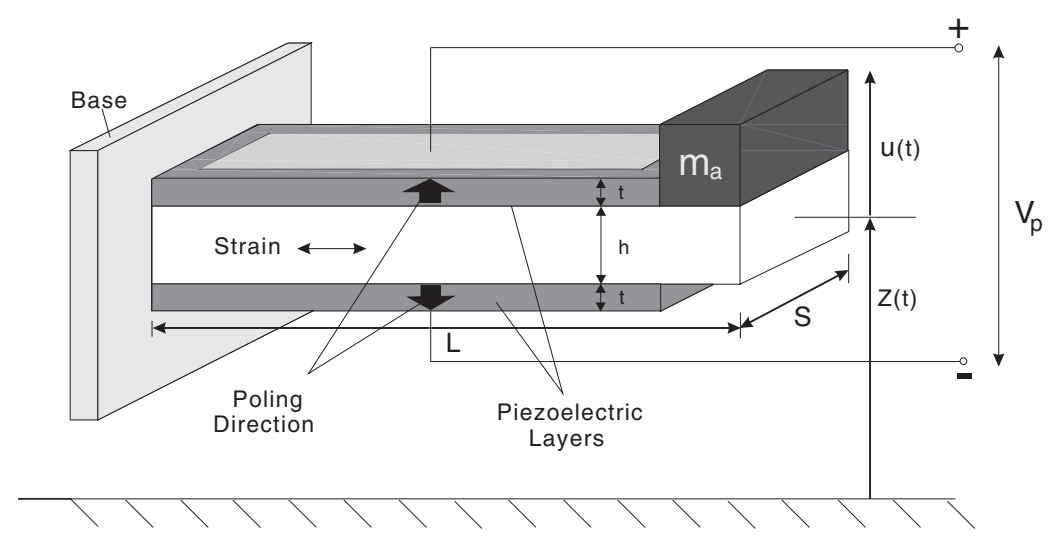

Figure 2. A common piezoelectric-based power generator: a cantilever triple-layer bender operated in the $\{3-1\}$ mode. The base is excited with acceleration $\ddot{z}(t)$. 


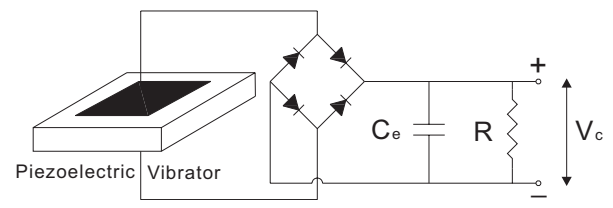

Figure 3. A typical AC-DC harvesting circuit.

A sinusoidal mechanical excitation

$$
F(t)=F_{0} \sin w t
$$

is applied to the system with $F_{0}$ the constant magnitude and $w\left(\right.$ in $\left.\mathrm{rad} \mathrm{s}^{-1}\right)$ the angular frequency of vibration. Note that in most vibration-based power harvesting systems the source of $F(t)$ is due to the excitation of the base with acceleration $\ddot{z}(t)$ as shown in figure 2.

Equation (3) is explained as follows. The rectifying bridge is open circuited if the voltage $\left|V_{p}\right|$ is smaller than the rectified voltage $V_{c}$. As a result, the current flowing into the circuit vanishes. On the other hand, when $\left|V_{p}\right|$ reaches $V_{c}$, the bridge conducts and the piezo voltage is kept equal to the rectified voltage; i.e. $\left|V_{p}\right|=V_{c}$. Finally, the conduction in the rectifier diodes is blocked again when the absolute value of the piezo voltage $\left|V_{p}(t)\right|$ starts decreasing.

As most applications require the output DC voltage $V_{c}$ to be stable, the common approach to achieving this is to assume that the filter capacitor $C_{e}$ is large enough so that the output voltage $V_{c}$ is essentially constant [28]. Specifically, $V_{c}(t)=$ $\left\langle V_{c}(t)\right\rangle+V_{\text {ripple }}$ where $\left\langle V_{c}(t)\right\rangle$ and $V_{\text {ripple }}$ are the average and ripple of $V_{c}(t)$, respectively. This average $\left\langle V_{c}(t)\right\rangle$ is independent of $C_{e}$ provided that the time constant $R C_{e}$ is much larger than the oscillating period of the generator [10]. The magnitude of $V_{\text {ripple }}$, however, depends on $C_{e}$ and is negligible for large $C_{e}$. Under this hypothesis, $V_{c}(t) \approx\left\langle V_{c}(t)\right\rangle$, and therefore in the following, we use $V_{c}$, instead of $\left\langle V_{c}(t)\right\rangle$, to represent the average of $V_{c}(t)$ for simplicity of notation.

To solve (1)-(4) under steady-state operation, we first determine the relation between the average value of the rectified voltage and displacement magnitude. From (2) and (3) the piezo voltage $V_{p}(t)$ varies proportionally with respect to the displacement $u(t)$ if the rectifying bridge is blocked and the outgoing piezoelectric current is zero. Therefore, solutions of $u(t)$ and $V_{p}(t)$ are assumed to take the following forms:

$$
u(t)=u_{0} \sin (w t-\theta), \quad V_{p}(t)=g(w t-\theta),
$$

where $u_{0}$ is the constant magnitude of displacement and $g(t)$ is a periodic function with period $2 \pi$ and $|g(t)| \leqslant V_{c}$. Let $T=\frac{2 \pi}{w}$ be the period of vibration, and $a$ and $b$ be two time instants $\left(b-a=\frac{T}{2}\right.$ ), such that the displacement $u$ goes from the minimum $-u_{0}$ to the maximum $u_{0}$. Assume that $\dot{V}_{p} \geqslant 0$ during the semi-period from $a$ to $b$. It follows that $\int_{a}^{b} \dot{V}_{p}(t) \mathrm{d} t=V_{c}-\left(-V_{c}\right)=2 V_{c}$. Note that $C_{e} \dot{V}_{c}(t)+\frac{V_{c}}{R}=0$ for $a<t<t^{*}$ during which the piezo voltage $\left|V_{p}\right|<V_{c}$ and the rectifier conducts when $t^{*} \leqslant t<b$. This gives from (3)

$$
\int_{a}^{b} I(t) \mathrm{d} t=\frac{T}{2} \frac{V_{c}}{R}
$$

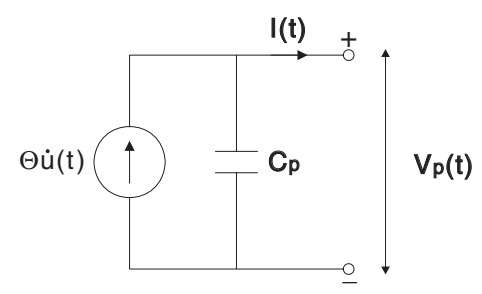

Figure 4. An equivalent circuit for the uncoupled model.

since the average current flowing through the capacitance $C_{e}$ is zero; i.e. $\int_{a}^{b} C_{e} \dot{V}_{c}(t) \mathrm{d} t=0$ for steady-state operation. The integration of (2) from time $a$ to $b$ is therefore

$$
-2 \Theta u_{0}+2 C_{p} V_{c}=-\frac{T}{2} \frac{V_{c}}{R},
$$

or

$$
V_{c}=\frac{w \Theta R}{w C_{p} R+\frac{\pi}{2}} u_{0} .
$$

Notice that (7) is identical to that derived by $[10,28]$.

The average harvested power can also be obtained in terms of the magnitude of displacement

$$
P=\frac{V_{c}^{2}}{R}=\frac{w^{2} \Theta^{2} R}{\left(w C_{p} R+\frac{\pi}{2}\right)^{2}} u_{0}^{2} .
$$

Thus, we need to find out $u_{0}$ to determine $V_{c}$ and $P$. There are two approaches in the literature for estimating this [10, 28]. We propose here another method for determining $u_{0}$, and show that this new estimation is more accurate than the other two in section 4. Before showing that, we introduce the following non-dimensionless parameters which will be used to simplify the analysis

$$
\begin{gathered}
w_{n}=\sqrt{\frac{K}{M}}, \quad k_{e}^{2}=\frac{\Theta^{2}}{K C_{p}}, \quad \zeta=\frac{\eta}{2 \sqrt{K M}}, \\
\Omega=\frac{w}{w_{n}}, \quad r=C_{p} w_{n} R,
\end{gathered}
$$

where $w_{n}$ is the natural frequency of short circuit, $k_{e}^{2}$ is the alternative electromechanical coupling coefficient ${ }^{2}, \zeta$ is the damping ratio and $\Omega$ and $r$ are the normalized frequency and electric resistance. Finally, there are two resonances for the system since the piezoelectric structure exhibits both short circuit and open circuit stiffness. They are defined by

$$
\Omega_{\mathrm{sc}}=1, \quad \Omega_{\mathrm{oc}}=\sqrt{1+k_{e}^{2}},
$$

where $\Omega_{\mathrm{sc}}$ and $\Omega_{\mathrm{oc}}$ are the frequency ratios of short circuit and open circuit, respectively. Note that the frequency shift is pronounced if the coupling factor $k_{e}^{2}$ is large.

\subsection{Uncoupled analysis}

Piezoelectric devices are frequently modeled as the current source in parallel with their internal electrode capacitance $C_{p}$ as shown in figure $4[6,7,13,27,28]$. This model is based on

\footnotetext{
2 The definition of $k_{e}^{2}$ here is slightly different from that used by [21].
} 
the assumption that the internal current source of the generator is independent of the impedance of the external load. This is equivalent to assuming that the coupling is very weak and the term $\Theta V_{p}$ can be dropped from (1). As a result, the governing equations (1) and (2) are simplified to be

$$
\begin{gathered}
M \ddot{u}(t)+\eta \dot{u}(t)+K u(t)=F(t), \\
-\Theta \dot{u}(t)+C_{p} \dot{V}_{p}(t)=-I(t) .
\end{gathered}
$$

As the displacement $u(t)$ can be solved independently from (11) using a simple harmonic analysis, $\Theta \dot{u}(t)$ can be treated as the known current source shown schematically in figure 4 . The rectified voltage $V_{c}$ and the average harvested power $P$ are therefore determined by (7) and (8). Finally, the normalized displacement $\bar{u}_{0}$, voltage $\bar{V}_{c}$ and power $\bar{P}$ in terms of non-dimensionless parameters ( 9 ) are described by

$$
\begin{gathered}
\bar{u}_{0}=\frac{u_{0}}{\frac{F_{0}}{K}}=\frac{1}{\left\{4 \zeta^{2} \Omega^{2}+\left(1-\Omega^{2}\right)^{2}\right\}^{\frac{1}{2}}}, \\
\bar{V}_{c}=\frac{V_{c}}{\frac{F_{0}}{\Theta}}=\left(\frac{r \Omega}{r \Omega+\frac{\pi}{2}}\right) \frac{k_{e}^{2}}{\left\{4 \zeta^{2} \Omega^{2}+\left(1-\Omega^{2}\right)^{2}\right\}^{\frac{1}{2}}}, \\
\bar{P}=\frac{P}{\frac{F_{0}^{2}}{w_{n} M}}=\frac{1}{\left(r \Omega+\frac{\pi}{2}\right)^{2}} \frac{k_{e}^{2} \Omega^{2} r}{\left\{4 \zeta^{2} \Omega^{2}+\left(1-\Omega^{2}\right)^{2}\right\}} .
\end{gathered}
$$

\subsection{In-phase analysis}

The uncoupled model assumes that the electromechanical coupling is very weak or the vibration amplitude is independent of the equivalent resistive load $R$. If the coupling is not so weak, Guyomar et al [10] have provided a new approach for estimating the average harvested power. Indeed, they have assumed that the external forcing function and the velocity of the mass are in phase. Precisely, (5) is changed to

$$
F(t)=F_{0} \sin w t, \quad \dot{u}(t)=u_{0} w \sin w t .
$$

As the derivation of the harvested power can be found in [10], we here only list their main results for future comparison. The normalized displacement $\bar{u}_{0}$, voltage $\bar{V}_{c}$ and power $\bar{P}$ are summarized in terms of the non-dimensionless system parameters

$$
\begin{gathered}
\bar{u}_{0}=\frac{u_{0}}{\frac{F_{0}}{K}}=\frac{1}{\left\{2 \zeta+\frac{2 k_{e}^{2} r}{\left(r \Omega+\frac{\pi}{2}\right)^{2}}\right\} \Omega}, \\
\bar{V}_{c}=\frac{V_{c}}{\frac{F_{0}}{\Theta}}=\left(\frac{r}{r \Omega+\frac{\pi}{2}}\right) \frac{k_{e}^{2}}{\left\{2 \zeta+\frac{2 k_{e}^{2} r}{\left(r \Omega+\frac{\pi}{2}\right)^{2}}\right\}}, \\
\bar{P}=\frac{P}{\frac{F_{0}^{2}}{w_{n} M}}=\frac{1}{\left(r \Omega+\frac{\pi}{2}\right)^{2}} \frac{k_{e}^{2} r}{\left\{2 \zeta+\frac{2 k_{e}^{2} r}{\left(r \Omega+\frac{\pi}{2}\right)^{2}}\right\}^{2}} .
\end{gathered}
$$

\subsection{Analytic analysis}

For a non-piezoelectric mechanical structure vibrating around resonance, the in-phase assumption between $F(t)$ and $\dot{u}(t)$ is a fairly reasonable approximation in the case of low damping. However, we are not aware whether this assumption still holds when non-small electromechanical coupling is taken into account. Hence, it is worth investigating this in detail here.

Let (1) be multiplied by $\dot{u}(t)$ and (2) be multiplied by $V_{p}(t)$. Integration of the addition of these two equations from time $a$ to $b$ gives the equation of the energy balance

$$
\begin{gathered}
\int_{a}^{b} F(t) \dot{u}(t) \mathrm{d} t=\int_{a}^{b} \eta \dot{u}^{2}(t) \mathrm{d} t+\int_{a}^{b} V_{p}(t) I(t) \mathrm{d} t \\
\quad+\left.\frac{1}{2} M \dot{u}^{2}(t)\right|_{a} ^{b}+\left.\frac{1}{2} K u^{2}(t)\right|_{a} ^{b}+\left.\frac{1}{2} C_{p} V_{p}^{2}(t)\right|_{a} ^{b} .
\end{gathered}
$$

Suppose that $F(t), u(t)$ and $V_{p}(t)$ are given by (4) and (5). Let $T=\frac{2 \pi}{w}$ and $a$ and $b$ be two time instants $\left(b-a=\frac{T}{2}\right)$ such that the displacement $u$ goes from the minimum $-u_{0}$ to the maximum $u_{0}$. The balance of energy (20) in this case becomes

$$
\int_{a}^{b} F(t) \dot{u}(t) \mathrm{d} t=\int_{a}^{b} \eta \dot{u}^{2}(t) \mathrm{d} t+\int_{a}^{b} V_{p}(t) I(t) \mathrm{d} t .
$$

We assume that $\dot{V}_{p} \geqslant 0$ during this semi-period from $a$ to $b$. Note that $C_{e} \dot{V}_{c}(t)+\frac{V_{c}}{R}=0$ for $a<t<$ $t^{*}$ during which the piezo voltage $\left|V_{p}\right|<V_{c}$. This also gives $\left(C_{e} \dot{V}_{c}(t)+\frac{V_{c}}{R}\right) V_{c}=0$ for $a<t<t^{*}$. The rectifier conducts later when the piezo voltage $V_{p}$ reaches the rectified voltage $V_{c}$, and from (3) $V_{p}(t) I(t)=$ $V_{c}\left(C_{e} \dot{V}_{c}(t)+\frac{V_{c}}{R}\right)$ during the conduction $t^{*} \leqslant t<b$. These arguments listed above suggest

$$
\int_{a}^{b} I(t) V_{p}(t) \mathrm{d} t=\frac{V_{c}^{2}}{R} \frac{T}{2}
$$

for steady-state operation. Next, substituting (4) and (5) into the equation of energy balance (21) results in

$$
\frac{\pi}{2} \eta w u_{0}^{2}+\frac{\pi}{w} \frac{V_{c}^{2}}{R}=\frac{\pi}{2} F_{0} u_{0} \sin \theta .
$$

Right now we have two equations (7) and (23) and three unknowns $u_{0}, V_{c}$ and $\theta$. We need a third one to solve them. From (2), we have

$$
\Theta \dot{V}_{p}(t)=\frac{\Theta}{C_{p}}[-I(t)+\Theta \dot{u}(t)] .
$$

Differentiating (1) with respect to time $t$ and substituting (24) into it, we find

$$
\begin{aligned}
& M \frac{\mathrm{d}}{\mathrm{d} t} \ddot{u}(t)+\eta \frac{\mathrm{d}}{\mathrm{d} t} \dot{u}(t)+\left(K+\frac{\Theta^{2}}{C_{p}}\right) \frac{\mathrm{d}}{\mathrm{d} t} u(t)-\frac{\Theta}{C_{p}} I(t) \\
& =\frac{\mathrm{d}}{\mathrm{d} t} F(t) .
\end{aligned}
$$

Integrating (25) with respect to time $t$ from $a$ to $b$ and using (5) and (6) provides

$\left(K-M w^{2}+\frac{\Theta^{2}}{C_{p}}\right) u_{0}-\frac{\pi \Theta}{2 C_{p} w R} V_{c}=F_{0} \cos \theta$.

Finally, we are in a position to determine $u_{0}$ in terms of system parameters. Combining both (23) and (26) gives

$$
\begin{aligned}
& \left\{\eta w u_{0}+\frac{2}{w R} \frac{V_{c}^{2}}{u_{0}}\right\}^{2} \\
& +\left\{\left(K-M w^{2}+\frac{\Theta^{2}}{C_{p}}\right) u_{0}-\frac{\pi \Theta}{2 C_{p} w R} V_{c}\right\}^{2}=F_{0}^{2}
\end{aligned}
$$


As the magnitude of displacement $u_{0}$ is related to the rectified voltage $V_{c}$ by (7), the above equation (27) can be further simplified to find $u_{0}$. The result is

$u_{0}=\frac{F_{0}}{\left\{\left(\eta w+\frac{2 w \Theta^{2} R}{\left(C_{p} w R+\frac{\pi}{2}\right)^{2}}\right)^{2}+\left(K-w^{2} M+\frac{w \Theta^{2} R}{C_{p} w R+\frac{\pi}{2}}\right)^{2}\right\}^{\frac{1}{2}}}$.

The following summarizes our main findings:

$$
\begin{aligned}
\bar{u}= & \frac{u_{0}}{\frac{F_{0}}{K}} \\
= & \frac{1}{\left\{\left(2 \zeta+\frac{2 k_{e}^{2} r}{\left(r \Omega+\frac{\pi}{2}\right)^{2}}\right)^{2} \Omega^{2}+\left(1-\Omega^{2}+\frac{\Omega k_{e}^{2} r}{r \Omega+\frac{\pi}{2}}\right)^{2}\right\}^{\frac{1}{2}}}, \\
\bar{V}_{c}= & \frac{V_{c}}{\frac{F_{0}}{\Theta}}=\left(\frac{r \Omega}{r \Omega+\frac{\pi}{2}}\right) \\
& \times \frac{k_{e}^{2}}{\left\{\left(2 \zeta+\frac{2 k_{e}^{2} r}{\left(r \Omega+\frac{\pi}{2}\right)^{2}}\right)^{2} \Omega^{2}+\left(1-\Omega^{2}+\frac{\Omega k_{e}^{2} r}{r \Omega+\frac{\pi}{2}}\right)^{2}\right\}^{\frac{1}{2}}}, \\
\bar{P}= & \frac{P}{F_{0}^{2}}=\frac{1}{\left(r \Omega+\frac{\pi}{2}\right)^{2}} k_{e}^{2} \Omega^{2} r \\
& \times \frac{2 k_{e}^{2} r}{\left\{\left(2 \zeta+\frac{\pi}{\left(r \Omega+\frac{\pi}{2}\right)^{2}}\right)^{2} \Omega^{2}+\left(1-\Omega^{2}+\frac{\Omega k_{e}^{2} r}{r \Omega+\frac{\pi}{2}}\right)^{2}\right\}},
\end{aligned}
$$

where (30) is interpreted as follows. Suppose the source of the forcing function comes from the vibration of the base of the structure, then this gives $F_{0}=M A$ where $A$ is the magnitude of acceleration of the exciting base. It follows that the harvested average power per unit mass is described by

$$
\frac{P}{M}=\frac{A^{2}}{w_{n}} \bar{P}\left(r, \Omega, k_{e}, \zeta\right) .
$$

This shows that the harvested average power per unit mass depends on the characteristics of the input vibration (frequency $\Omega$ and acceleration $A$ ), the normalized electric resistance $r$, the short circuit resonance $w_{n}$, the mechanical damping ratio $\zeta$, and the overall electromechanical coupling coefficient $k_{e}^{2}$ of the system. Thus, the scheme to optimize the power either by tuning the electric resistance, selecting suitable operation points or adjusting the coupling coefficient by optimal structural design can be guided completely by (30).

\section{Optimal resistance and power}

Suppose $\Omega, k_{e}$ and $\zeta$ are fixed. The design criterion for reaching the maximal power flow under steady-state operation can be obtained by tuning the load impedance according to

$$
\left.\frac{\partial}{\partial r} \bar{P}\left(r, \Omega, k_{e}, \zeta\right)\right|_{\Omega, k_{e}, \zeta}=0 .
$$

We use the notation $r^{\text {opt }}$ to represent the solution of (31), and $r^{\text {opt }}=r^{\mathrm{opt}}\left(\Omega, k_{e}, \zeta\right)$ in general. Besides, the superscript 'opt' denotes functions evaluated at the optimal load resistance $r^{\text {opt }}$. For example,

$$
\bar{P}^{\mathrm{opt}}=\bar{P}\left(r^{\mathrm{opt}}, \Omega, k_{e}, \zeta\right) ; \text { etc. }
$$

The selection of the suitable operation frequency is also important to maximize the average harvested power, and we will discuss this in section 3.3.

\subsection{Uncoupled analysis}

Substituting (15) into (31), we find the optimal load is

$$
r^{\mathrm{opt}}=\frac{\pi}{2 \Omega} \quad \text { or } \quad R^{\mathrm{opt}}=\frac{\pi}{2 C_{p} w} .
$$

It follows from (13), (14) and (15) that the normalized displacement, voltage and power evaluated at the optimal load are

$$
\begin{gathered}
\bar{u}_{0}^{\mathrm{opt}}=\frac{u_{0}^{\mathrm{opt}}}{\frac{F_{0}}{K}}=\frac{1}{\left\{4 \zeta^{2} \Omega^{2}+\left(1-\Omega^{2}\right)^{2}\right\}^{\frac{1}{2}}}, \\
\bar{V}_{c}^{\mathrm{opt}}=\frac{V_{c}^{\mathrm{opt}}}{\frac{F_{0}}{\Theta}}=\frac{1}{2} \frac{k_{e}^{2}}{\left\{4 \zeta^{2} \Omega^{2}+\left(1-\Omega^{2}\right)^{2}\right\}^{\frac{1}{2}}}, \\
\bar{P}^{\mathrm{opt}}=\frac{P^{\mathrm{opt}}}{\frac{F_{0}^{2}}{w_{n} M}}=\frac{1}{2 \pi} \frac{k_{e}^{2} \Omega}{\left\{4 \zeta^{2} \Omega^{2}+\left(1-\Omega^{2}\right)^{2}\right\}} .
\end{gathered}
$$

In the uncoupled model the optimal harvested power flow can be achieved by tuning the load impedance to match the internal impedance of the piezoelectric generator, i.e. $R^{\mathrm{opt}}=\frac{\pi}{2 C_{p} w}$. In addition, the rectified voltage $V_{c}^{\mathrm{opt}}=\frac{1}{2} V_{\mathrm{oc}}$ where $V_{\mathrm{oc}}$ is the maximum voltage at the open circuit condition for the uncoupled model [28].

\subsection{In-phase analysis}

Lefeuvre et al [19] have questioned the soundness of the uncoupled model and proposed a modified estimation of the optimal load based on the in-phase assumption. The results are classified according to the inequality of $\frac{k_{e}^{2}}{\zeta}-2 \pi \Omega$ and are listed below for future comparison.

Case 1: $\frac{k_{e}^{2}}{\zeta}-2 \pi \Omega \leqslant 0$. The optimal normalized load, displacement, voltage and power evaluated at the optimal condition are

$$
r_{o}^{\mathrm{opt}}=\frac{\pi}{2 \Omega}
$$

$$
\begin{gathered}
\bar{u}_{0}^{\mathrm{opt}}=\frac{u_{0}^{\mathrm{opt}}}{\frac{F_{0}}{K}}=\frac{1}{\left\{2 \zeta \Omega+\frac{k_{e}^{2}}{\pi}\right\}}, \\
\bar{V}_{c}^{\mathrm{opt}}=\frac{V_{c}^{\mathrm{opt}}}{\frac{F_{0}}{\Theta}}=\frac{1}{2} \frac{k_{e}^{2}}{\left\{2 \zeta \Omega+\frac{k_{e}^{2}}{\pi}\right\}}, \\
\bar{P}^{\mathrm{opt}}=\frac{P^{\mathrm{opt}}}{\frac{F_{0}^{2}}{w_{n} M}}=\frac{1}{2 \pi} \frac{k_{e}^{2} \Omega}{\left\{2 \zeta \Omega+\frac{k_{e}^{2}}{\pi}\right\}^{2}} .
\end{gathered}
$$

Note that the optimal resistance (36) is the same as (32), and the optimal power in (39) is close to (35) provided that $\frac{k_{e}^{2}}{\zeta} \ll 1$ and $\Omega \approx 1$. 
Case 2: $\frac{k_{e}^{2}}{\zeta}-2 \pi \Omega \geqslant 0$. Suppose the electromechanical coupling factor $k_{e}^{2}$ is large and the damping is small. The optimal resistance $r_{o}^{\text {opt }}$ in (36) turns out to be the one minimizing the power flow. There are two new optimal resistances called $r_{a}^{\mathrm{opt}}$ and $r_{b}^{\mathrm{opt}}$ to maximize the power in this case, and the corresponding normalized displacement, voltage and power are

$r_{a}^{\mathrm{opt}}=\frac{1}{2 \Omega^{2}}\left\{\left(\frac{k_{e}^{2}}{\zeta}-\pi \Omega\right)-\sqrt{\left(\frac{k_{e}^{2}}{\zeta}-\pi \Omega\right)^{2}-(\pi \Omega)^{2}}\right\}$

$r_{b}^{\mathrm{opt}}=\frac{1}{2 \Omega^{2}}\left\{\left(\frac{k_{e}^{2}}{\zeta}-\pi \Omega\right)+\sqrt{\left(\frac{k_{e}^{2}}{\zeta}-\pi \Omega\right)^{2}-(\pi \Omega)^{2}}\right\}$.

$\left.\bar{u}_{0}^{\mathrm{opt}}\right|_{r=r_{a}^{\mathrm{opt}}}=\left.\frac{u_{0}^{\mathrm{opt}}}{\frac{F_{0}}{K}}\right|_{r=r_{a}^{\mathrm{opt}}}=\left.\bar{u}_{0}^{\mathrm{opt}}\right|_{r=r_{b}^{\mathrm{opt}}}=\left.\frac{u_{0}^{\mathrm{opt}}}{\frac{F_{0}}{K}}\right|_{r=r_{b}^{\mathrm{opt}}}=\frac{1}{4 \zeta \Omega}$,

$\left.\bar{V}_{c}^{\mathrm{opt}}\right|_{r=r_{a}^{\mathrm{opt}}}=\left.\frac{V_{c}^{\mathrm{opt}}}{\frac{F_{0}}{\Theta}}\right|_{r=r_{a}^{\mathrm{opt}}}=\frac{k_{e}^{2}}{8 \zeta \Omega}\left\{1-\sqrt{1-\frac{2 \pi \Omega}{\frac{k_{e}^{2}}{\zeta}}}\right\}$,

$\left.\bar{V}_{c}^{\mathrm{opt}}\right|_{r=r_{b}^{\mathrm{opt}}}=\left.\frac{V_{c}^{\mathrm{opt}}}{\frac{F_{0}}{\Theta}}\right|_{r=r_{b}^{\mathrm{opt}}}=\frac{k_{e}^{2}}{8 \zeta \Omega}\left\{1+\sqrt{1-\frac{2 \pi \Omega}{\frac{k_{e}^{2}}{\zeta}}}\right\}$,

$\left.\bar{P}^{\mathrm{opt}}\right|_{r=r_{a}^{\mathrm{opt}}}=\left.\frac{P^{\mathrm{opt}}}{\frac{F_{0}^{2}}{w_{n} M}}\right|_{r=r_{a}^{\mathrm{opt}}}=\left.\bar{P}^{\mathrm{opt}}\right|_{r=r_{b}^{\mathrm{opt}}}=\left.\frac{P^{\mathrm{opt}}}{\frac{F_{0}^{2}}{w_{n} M}}\right|_{r=r_{b}^{\mathrm{opt}}}$

$=\frac{1}{16 \zeta}$

Note that $r_{a}^{\text {opt }}<r_{o}^{\text {opt }}<r_{b}^{\text {opt }}$. It is interesting to see that the harvested average power has two identical maxima and depends only on the internal damping of the generator. Lefeuvre et al [19] have interpreted the appearance of two optimal resistances as characteristic of a strongly coupled system. However, this has to be taken with caution since there are always two optimal loads for each applied frequency in the in-phase model provided that $\frac{k_{e}^{2}}{\zeta}-2 \pi \Omega \geqslant 0$. We will discuss it in section 3.3.

\subsection{Analytic analysis}

The power derived from the analytic analysis is given by (30). Although it extensively describes the characteristics of the harvesting system, the complicated nature of (30) makes it difficult to derive the closed form solution of the optimal resistance from (31). Alternatively, we discuss the functional behaviour of (30) according to the different ranges of the parameter $\frac{k_{\rho}^{2}}{\zeta}$. We study the small and medium ranges of $\frac{k_{\rho}^{2}}{\zeta}$ in sections 4.1 and 4.2 , respectively. Here we provide an analysis to show that the harvested power can be maximized at two different electrical loads at the respective operating points provided that $\frac{k_{e}^{2}}{\zeta} \gg 1$. However, we are not aware under exactly what condition there exist two optimal pairs. But if the AC-DC harvesting circuit shown in figure 3 is changed to an $\mathrm{AC}$ circuit, it can be shown that two optimal pairs appear whenever

$$
\frac{k_{e}^{2}}{\zeta} \geqslant 4(\zeta+1)
$$

Returning to the standard AC-DC circuit, our results of numerous numerical simulations suggest

$$
\frac{k_{e}^{2}}{\zeta} \geqslant 10
$$

as the rule of thumb for the appearance of two optimal pairs, and we will use it as a criterion for designing a strongly coupled electromechanical system.

Case 1: Short circuit resonance. The power $P$ in (30) for $\Omega=\Omega_{\mathrm{sc}}=1$ can be expressed as

$$
\frac{P}{\frac{F_{0}^{2}}{w_{n} M}}=\frac{1}{\zeta} \frac{r x}{\left\{4\left[\left(r+\frac{\pi}{2}\right)+\frac{r x}{\left(r+\frac{\pi}{2}\right)}\right]^{2}+r^{2} x^{2}\right\}},
$$

where $x=\frac{k_{e}^{2}}{\zeta}$. Suppose the parameter $x \gg 1$. To optimize the power in (47), $r$ has to be small or proportional to the inverse of $x$; otherwise, the power will tend to zero for non-small $r$ while $x$ remains extremely large. This gives

$$
\frac{P}{\frac{F_{0}^{2}}{w_{n} M}} \approx \frac{1}{\zeta} \frac{r x}{\left\{4\left[\frac{\pi}{2}+\frac{2}{\pi} r x\right]^{2}+r^{2} x^{2}\right\}}
$$

for small $r$ and $x \gg 1$. The optimal power flow can be obtained by differentiating (48) with respect to $r$. It follows that

$r_{\mathrm{sc}}^{\mathrm{opt}} \approx\left(\frac{\pi^{2}}{\sqrt{16+\pi^{2}}}\right) \frac{1}{\frac{k_{e}^{2}}{\zeta}} \quad$ if $x=\frac{k_{e}^{2}}{\zeta} \gg 1$.

The corresponding normalized displacement, voltage and power are

$$
\begin{gathered}
\bar{u}_{0}^{\mathrm{opt}}=\frac{u_{0}^{\mathrm{opt}}}{\frac{F_{0}}{K}} \approx \sqrt{\frac{16+\pi^{2}}{32+2 \pi^{2}+8 \sqrt{16+\pi^{2}}}} \frac{1}{2 \zeta}, \\
\bar{V}_{c}^{\mathrm{opt}}=\frac{V_{c}^{\mathrm{opt}}}{\frac{F_{0}}{\Theta}} \approx \frac{\pi}{\sqrt{32+2 \pi^{2}+8 \sqrt{16+\pi^{2}}}}, \\
\bar{P}^{\mathrm{opt}}=\frac{P_{\text {opt }}}{\frac{F_{0}^{2}}{w_{n} M}} \approx\left(\frac{1}{8+2 \sqrt{16+\pi^{2}}}\right) \frac{1}{\zeta} .
\end{gathered}
$$

Case 2: Open circuit resonance. We set $x=\frac{k_{e}^{2}}{\zeta}$ again. The power $P$ in (30) for the applied frequency operated at $\Omega_{\mathrm{oc}}=\sqrt{1+k_{e}^{2}}$ can be expressed as

$$
\frac{P}{\frac{F_{0}^{2}}{w_{n} M}}=\frac{1}{\zeta} \frac{r x \Omega_{\mathrm{oc}}^{2}}{\left\{4\left[\left(r \Omega_{\mathrm{oc}}+\frac{\pi}{2}\right)+\frac{r x}{\left(r \Omega_{\mathrm{oc}}+\frac{\pi}{2}\right)}\right]^{2} \Omega_{\mathrm{oc}}^{2}+\frac{\pi^{2}}{4} x^{2}\right\}} .
$$

To estimate the optimal power in (53) for the case of $x \gg 1$, $r$ has to be proportional to $x$ by examining the term $r x$ in the numerator and $\frac{\pi^{2}}{4} x^{2}$ in the denominator. This shows that $r$ has to be large to maximize (53). Hence, we may rewrite (53) as

$$
\frac{P}{\frac{F_{0}^{2}}{w_{n} M}} \approx \frac{1}{\zeta} \frac{r x \Omega_{\mathrm{oc}}^{2}}{\left\{4\left[r \Omega_{\mathrm{oc}}+\frac{x}{\Omega_{\mathrm{oc}}}\right]^{2} \Omega_{\mathrm{oc}}^{2}+\frac{\pi^{2}}{4} x^{2}\right\}}
$$


Table 1. The relation between the system parameters $k_{e}^{2}$ and $\zeta$ and the normalized electric resistance, displacement, voltage, current and power designed at the maximal power flow operated at the short circuit $\left(\Omega_{\mathrm{sc}}\right)$ and open circuit $\left(\Omega_{\mathrm{oc}}\right)$ resonances. The normalized current is defined as $\bar{I}=\frac{\bar{V}_{c}}{r}$. Note that the condition $\frac{k_{e}^{2}}{\zeta} \gg 1$ is implied in the analysis.

\begin{tabular}{lll}
\hline Optimal conditions & $\Omega_{\mathrm{sc}}$ & $\Omega_{\mathrm{oc}}$ \\
\hline Resistance & $r_{\mathrm{sc}}^{\mathrm{opt}} \propto \frac{1}{\frac{k_{e}^{2}}{\zeta}}<r_{\mathrm{oc}}^{\mathrm{opt}} \propto \frac{1}{\left(1+k_{e}^{2}\right)} \frac{k_{e}^{2}}{\zeta}$ \\
Displacement & $\bar{u}_{0}^{\mathrm{opt}} \propto \frac{1}{\zeta}>\bar{u}_{0}^{\mathrm{opt}} \propto \frac{1}{\zeta\left(\sqrt{1+k_{e}^{2}}\right)}$ \\
Voltage & $\bar{V}_{c}^{\mathrm{opt}} \propto 1<\bar{V}_{c}^{\mathrm{opt}} \propto \frac{1}{\sqrt{1+k_{e}^{2}}} \frac{k_{e}^{2}}{\zeta}$ \\
Current & $\bar{I}^{\mathrm{opt}} \propto \frac{k_{e}^{2}}{\zeta}>\bar{I}^{\mathrm{opt}} \propto \sqrt{1+k_{e}^{2}}$ \\
Power & $\bar{P}^{\mathrm{opt}} \propto \frac{1}{\zeta}=\bar{P}^{\mathrm{opt}} \propto \frac{1}{\zeta}$ \\
\hline
\end{tabular}

provided $x \gg 1$. The optimal power flow can be obtained by differentiating (54) with respect to $r$. This gives

$$
r_{\mathrm{oc}}^{\mathrm{opt}} \approx \frac{\sqrt{16+\pi^{2}}}{4} \frac{\frac{k_{e}^{2}}{\zeta}}{1+k_{e}^{2}} \quad \text { if } x=\frac{k_{e}^{2}}{\zeta} \gg 1 .
$$

The corresponding normalized displacement, voltage and power are

$\bar{u}_{0}^{\mathrm{opt}}=\frac{u_{0}^{\mathrm{opt}}}{\frac{F_{0}}{K}} \approx \sqrt{\frac{16+\pi^{2}}{32+2 \pi^{2}+8 \sqrt{16+\pi^{2}}}} \frac{1}{2 \zeta \sqrt{1+k_{e}^{2}}}$,

$\bar{V}_{c}^{\mathrm{opt}}=\frac{V_{c}^{\mathrm{opt}}}{\frac{F_{0}}{\Theta}} \approx \sqrt{\frac{16+\pi^{2}}{32+2 \pi^{2}+8 \sqrt{16+\pi^{2}}}} \frac{k_{e}^{2}}{2 \zeta \sqrt{1+k_{e}^{2}}}$,

$\bar{P}^{\mathrm{opt}}=\frac{P^{\mathrm{opt}}}{\frac{F_{0}^{2}}{w_{n} M}} \approx\left(\frac{1}{8+2 \sqrt{16+\pi^{2}}}\right) \frac{1}{\zeta}$.

Discussions: In the case of large $\frac{k_{e}^{2}}{\zeta}$, we find that for each applied frequency there is only one optimal load to maximize the power output. In addition, we have shown that the harvested power has two identical peaks, but is optimized at different resistances and operation frequencies; i.e.

$$
\bar{P}\left(r_{\mathrm{sc}}^{\mathrm{opt}}, \Omega_{\mathrm{sc}}, k_{e}, \zeta\right)=\bar{P}\left(r_{\mathrm{oc}}^{\mathrm{opt}}, \Omega_{\mathrm{oc}}, k_{e}, \zeta\right)
$$

provided that $\frac{k_{e}^{2}}{\zeta} \gg 1$. These results are in contrast with those obtained by the in-phase assumption. There always exist two optimal resistances given by (40) and (41) for every frequency in the in-phase model whenever $\frac{k_{e}^{2}}{\zeta}-2 \pi \Omega \geqslant 0$ (see also figure 9(f)). Besides, the in-phase model predicts the identical displacement evaluated at two optimal loads (see (42)) while our analytic analysis predicts unequal peaks of displacement; c.f. (50) and (56). This result is crucial in the design of microscale power generators [5].

Next, (49) and (55) suggest $r_{\mathrm{sc}}^{\mathrm{opt}} \propto \frac{1}{\left(k_{e}^{2} / \zeta\right)} \ll 1$ while $r_{\mathrm{oc}}^{\mathrm{opt}} \leqslant \frac{1}{\zeta}$. Therefore, $r_{\mathrm{sc}}^{\mathrm{opt}}$ can be made as small as possible by increasing the electromechanical coupling coefficient $k_{e}^{2}$ while $r_{\mathrm{oc}}^{\mathrm{opt}}$ has an upper bound. Finally, table 1 summarizes the relation between the system parameters $k_{e}^{2}$ and $\zeta$ and the normalized load, displacement, voltage, current and power designed at the maximal power flow operated either at the short circuit $\left(\Omega_{\mathrm{sc}}\right)$ or open circuit $\left(\Omega_{\mathrm{oc}}\right)$ resonances.

\section{Comparisons}

We now show that in section 4.1 the various forms of power derived from different approaches are almost the same provided that the parameter $\frac{k_{e}^{2}}{\zeta}$ is small, and we will use examples including experimental validation to demonstrate that the discrepancies among these analyses become large when the parameter $\frac{k_{e}^{2}}{\zeta}$ is increasing. In section 4.3 we find that the average harvested power is maximized at two optimal loads operated at different frequencies in the case of large $\frac{k_{c}^{2}}{\zeta}$.

\subsection{Small $\frac{k_{e}^{2}}{\zeta}$}

Suppose that $k_{e}^{2} \ll 1$ and $\frac{k_{e}^{2}}{\zeta} \ll 1$. Thus, the shift in frequency from $\Omega_{\mathrm{sc}}$ to $\Omega_{\mathrm{oc}}$ is not pronounced for small $k_{e}^{2}$. Let the applied frequency ratio $\Omega$ be operated between $\Omega_{\mathrm{sc}}$ and $\Omega_{\mathrm{oc}}$. We may set

$$
\Omega^{2}=1+f k_{e}^{2}, \quad 0 \leqslant f \leqslant 1 .
$$

Set $x=\frac{k_{e}^{2}}{\zeta}$. The power $P$ derived from the analytic analysis in (30) can be expressed as

$$
\begin{aligned}
& \frac{P}{\frac{F_{0}^{2}}{w_{n} M}}=\frac{r}{4 \zeta\left(r \Omega+\frac{\pi}{2}\right)^{2}} \\
& \quad \times \frac{x}{\left\{\left(1+\frac{x r}{\left(r \Omega+\frac{\pi}{2}\right)^{2}}\right)^{2}+\frac{x^{2}}{4 \Omega^{2}}\left(-f+\frac{\Omega r}{r \Omega+\frac{\pi}{2}}\right)^{2}\right\}} \\
& \approx \frac{r x}{4 \zeta\left(r \Omega+\frac{\pi}{2}\right)^{2}}\{1+\mathrm{O}(x)\} \\
& \approx \frac{k_{e}^{2} r}{4 \zeta^{2}\left(r \Omega+\frac{\pi}{2}\right)^{2}}
\end{aligned}
$$

provided that $x \ll 1$ and $\Omega \approx \Omega_{\mathrm{sc}} \approx \Omega_{\mathrm{oc}} \approx 1$. The notation $\mathrm{O}(x)$ denotes the higher order terms which tend to zero as $x$ tends to zero. Comparing (59) with the power derived from the uncoupled assumption (15) and that from the in-phase assumption (19) justifies our assertion. Figures 5(a) and (b) are the normalized rectified voltage and average harvested power versus the normalized resistance around resonance in the case of $k_{e}=0.05$ and $\zeta=0.03$. The solid, dashed and long-dashed lines are results derived from the analytic, in-phase and uncoupled solutions. These three lines are almost coincident. We therefore conclude that the conventional uncoupled solution is suitable if $\frac{k_{e}^{2}}{\zeta} \ll 1$.

\subsection{Medium $\frac{k_{e}^{2}}{\zeta}$}

The discrepancies among these approaches become significant when the ratio $\frac{k_{e}^{2}}{\zeta}$ increases. For piezoelectric generators operated in the $\{3-1\}$ mode, $k_{e}^{2}$ can approach $\frac{e_{31}^{2}}{C_{11}^{k} \varepsilon_{33}^{\zeta}}$ if the structure is made up entirely of piezoelectric materials [5]. Most electromechanical structures are made up of both piezoelectric and non-piezoelectric materials. The factor $k_{e}^{2}$ is then usually less than the theoretical value. On the other hand, the coupling coefficient $k_{e}^{2}$ can approach its upper bound for micro-scale devices since the contribution of piezoelectric elements to the overall structural stiffness is significant in this 

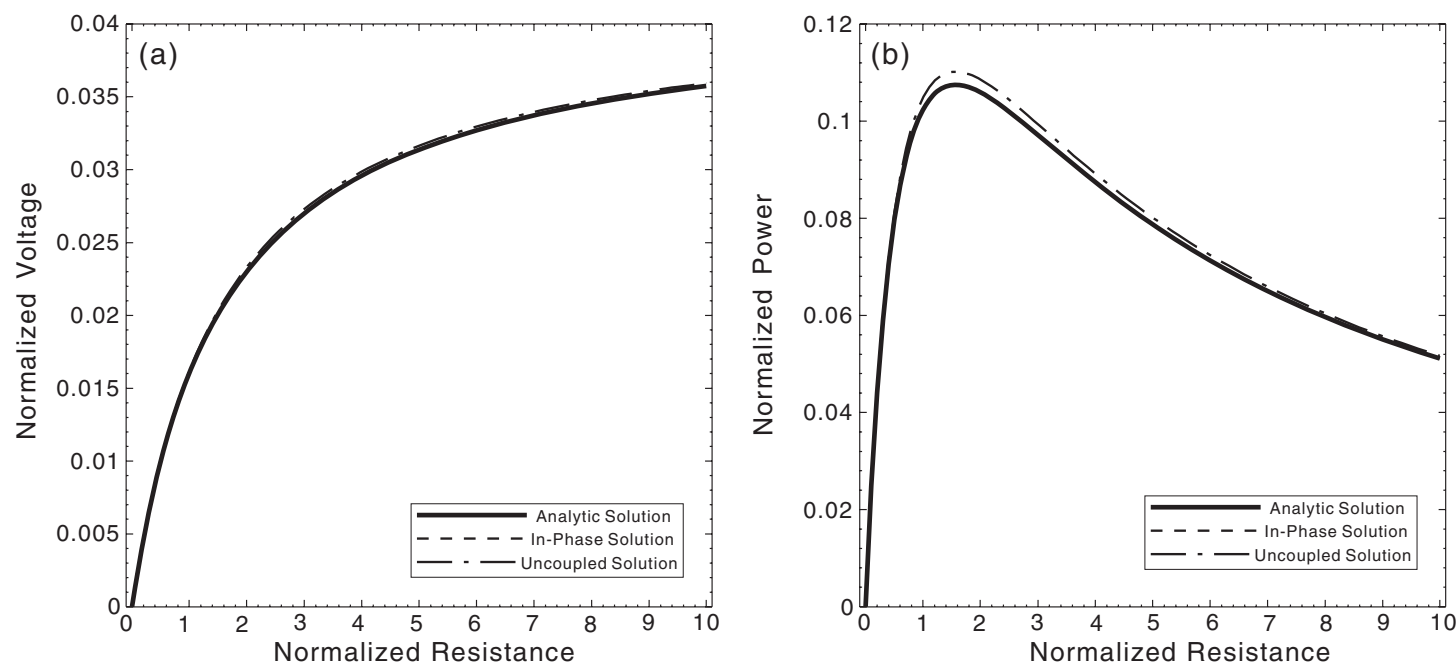

Figure 5. Comparisons in the case of small $\frac{k_{e}^{2}}{\zeta}$. We use $k_{e}=0.05$ and $\zeta=0.03\left(\frac{k_{e}^{2}}{\zeta}=0.083\right)$. (a) Normalized rectified voltage versus normalized resistance. (b) Normalized harvested power versus normalized resistance.

case [5]. We then investigate it in detail here. We assume $k_{e}=0.4$ and $\zeta=0.03\left(\frac{k_{e}^{2}}{\zeta}=5.33\right)$ in the following analysis.

The normalized displacement, voltage and power against the normalized electric resistance are plotted in figures 6(a)(c) operated at the short circuit resonance and in figures 6(d)(f) operated at the open circuit resonance. The long-dashed, dashed and solid lines are results calculated based on the uncoupled, in-phase and analytic solutions. As expected, predictions from the uncoupled analysis are far away from those predicted by either analytic or in-phase solutions since the electromechanical coupling is not small in this case. We then conclude that the uncoupled solution is not suitable for medium or even large $\frac{k_{e}^{2}}{\zeta}$.

Figure 6 also reveals substantial differences between our analytic and the in-phase analyses. We therefore use both simulation and experiment to determine which approaches predict more accurate behaviour of the energy harvesting system. Consider the numerical simulation first. Notice that (1) and (2) can be transformed to an equivalent $R L C$ circuit with $R=\frac{\eta}{\Theta^{2}}$ as resistance, $L=\frac{M}{\Theta^{2}}$ as inductance and $C=\frac{\Theta^{2}}{K}$ as capacitance. We use the software PSpice to simulate this equivalent circuit connected to the AC-DC harvesting circuit shown in figure 3 . The results are illustrated in figure 7 where we plot the normalized power versus electric resistance at short circuit and open circuit resonances. The numerical results are marked in figure 7 using open circles. Apparently, the numerical simulations favor results predicted based on our analytic solutions. In particular, our approach accurately predicts the optimal electric load resistance maximizing the average harvested power. The optimal load $r_{\mathrm{sc}}^{\mathrm{opt}}$ (analytic) $=$ 0.45 is smaller at $\Omega_{\mathrm{sc}}$ while $r_{\mathrm{oc}}^{\mathrm{opt}}$ (analytic) $=4.64$ is larger at $\Omega_{\mathrm{oc}}$. However, the optimal resistances predicted by the inphase solutions at $\Omega_{\mathrm{sc}}$ and $\Omega_{\mathrm{oc}}$ are very close: $r_{\mathrm{sc}}^{\mathrm{opt}}$ (in-phase) $=$ 1.57 and $r_{\mathrm{oc}}^{\mathrm{opt}}$ (in-phase) $=1.46$, and therefore are not suitable for the design.

Finally, we validate the analytic solution by our recent experiment. The specimen is a piezoelectric triple-layer bender with the overall dimension $40 \mathrm{~mm} \times 20 \mathrm{~mm} \times 0.36 \mathrm{~mm}(L \times$
$S \times(h+2 t))$ as shown similarly in figure 2 . The overall mass of the beam is $m_{p}+m_{b}=2.2509 \mathrm{~g}$ and an attached mass $m_{a}=0.4207 \mathrm{~g}$ is put at the tip. The measured open circuit and short circuit resonances are 52.9 and $53.7 \mathrm{~Hz}$, respectively. This gives a coupling factor $k_{e}$ around 0.17 . The mechanical damping ratio is measured at about 0.01 , and therefore $\frac{k_{e}^{2}}{\zeta}=2.89$. The applied acceleration is around $1.856 \mathrm{~m} \mathrm{~s}^{-2}$ and is slightly dependent on electrical resistances. The power harvesting circuit is chosen using the standard ACDC circuit illustrated in figure 3 . The values of rectified voltage $V_{c}$ are measured at the open circuit resonance for various electrical resistances and are marked in figure 8(a) using dark circles $\bullet$. The corresponding values of harvested power are also plotted against the various electrical resistances in figure 8 (b). The optimal electrical resistance can be determined from figure 8 (b) and is around $R_{\mathrm{exp}}^{\mathrm{opt}} \approx 200 \mathrm{k} \Omega$. The predicted results from the uncoupled, in-phase and analytic solutions are represented by long-dashed, dashed and solid lines in figure 8. As expected, the uncoupled solutions are not able to reflect the electrical performances of the system. The inphase solutions also overestimate the measured voltage and power, and underestimate the optimal load $\left(R_{\mathrm{in}-\text { phase }}^{\text {opt }} \approx 88 \mathrm{k} \Omega\right.$ ). On the other hand, the analytic solutions are close to the experimental observations and the predicted optimal load is around $R_{\text {analytic }}^{\text {opt }} \approx 210 \mathrm{k} \Omega$ which is pretty close to the measured one. The deviations between the experimental results and the analytic solutions are believed to be due to diode loss which has not been incorporated in the current analysis.

\subsection{Large $\frac{k_{e}^{2}}{\zeta}$}

The shift in frequency is significant if either the piezoelectric constant or the contribution of the piezoelectric element to the overall stiffness is large; i.e. $k_{e}^{2}$ is large. In particular, a piezoelectric power generator operated in the \{3-3\} (longitudinal) mode can have high coupling coefficient $k_{e}^{2}$ approaching $\frac{e_{33}^{2}}{C_{33}^{E} \varepsilon_{33}^{S}}$ if the piezoelectric element constitutes the whole structure [5]. Besides, if the mechanical damping 

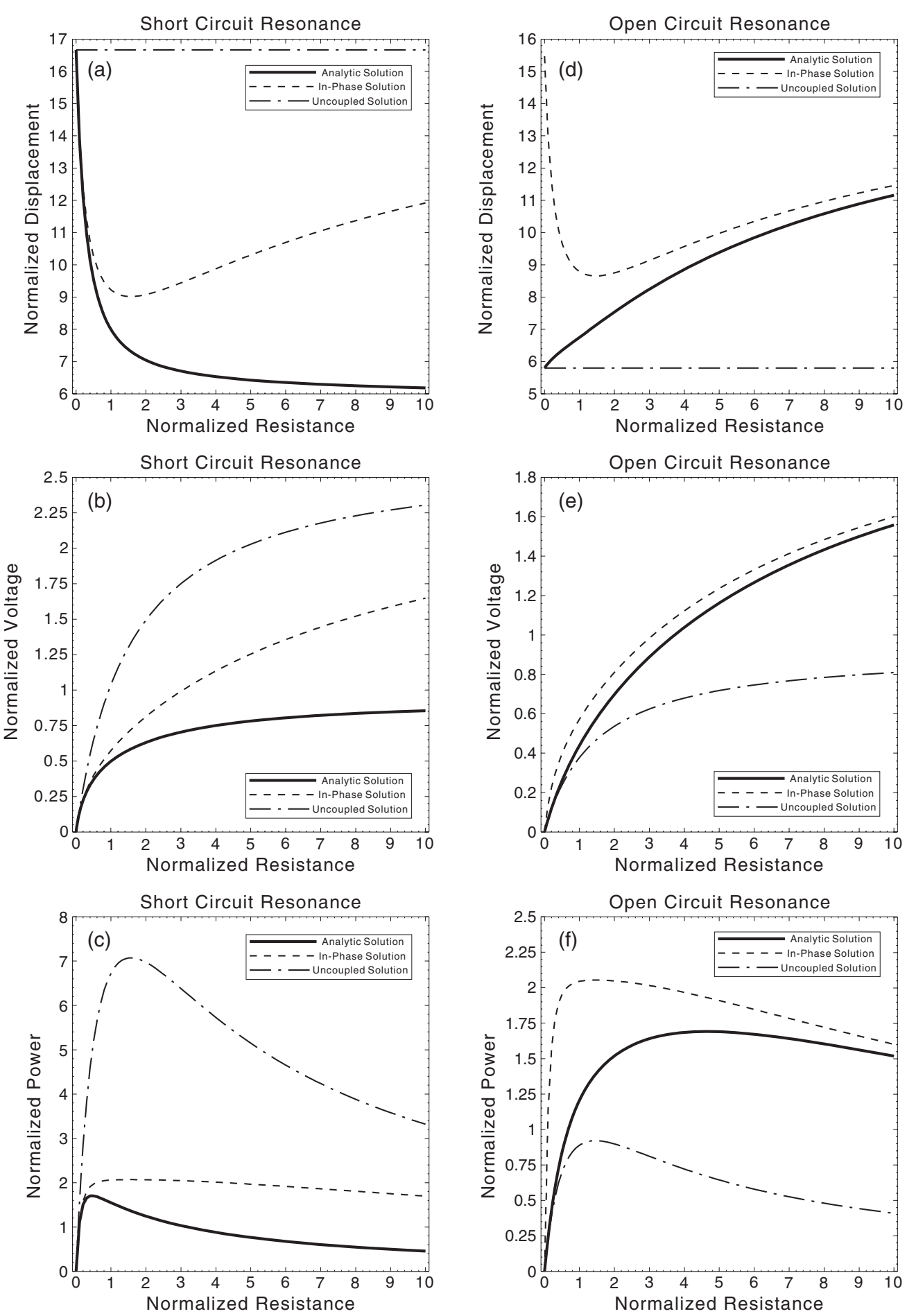

Figure 6. Comparisons for medium $\frac{k_{e}^{2}}{\zeta}$. We use $k_{e}=0.4$ and $\zeta=0.03\left(\frac{k_{e}^{2}}{\zeta}=5.33\right)$ : (a)-(c) are the normalized displacement, voltage and power versus normalized resistance operated at $\Omega_{\mathrm{sc}}$ while (d)-(f) are those operated at $\Omega_{\mathrm{oc}}$.

ratio is small, or the factor $\frac{k_{e}^{2}}{\zeta}$ is large, the selection of the correct operating frequency is very important for achieving the maximal power. Recently, much experimental effort has been made to fabricate small-scale piezoelectric cantilever beams with interdigitated electrodes on the beam surface to produce the $\{3-3\}$ mode using various materials $[12,13,47]$. Some of their chosen materials such as PZN-PT and PMN-PT relaxor ferroelectrics can have even higher piezoelectric constants than conventional PZT. As a result, the shift in resonance is expected to be pronounced due to large $k_{e}^{2}$ in these micro-scale devices, and we study this effect on power harvesting now.

We assume $k_{e}=1.14$ and $\zeta=0.03$. This gives $\Omega_{\mathrm{sc}}=1, \Omega_{\mathrm{oc}}=1.52, \frac{k_{\rho}^{2}}{\zeta}=43.3$. As illustrated in section 4.2 , results predicted from the uncoupled analysis are 
Analysis of power output for piezoelectric energy harvesting systems
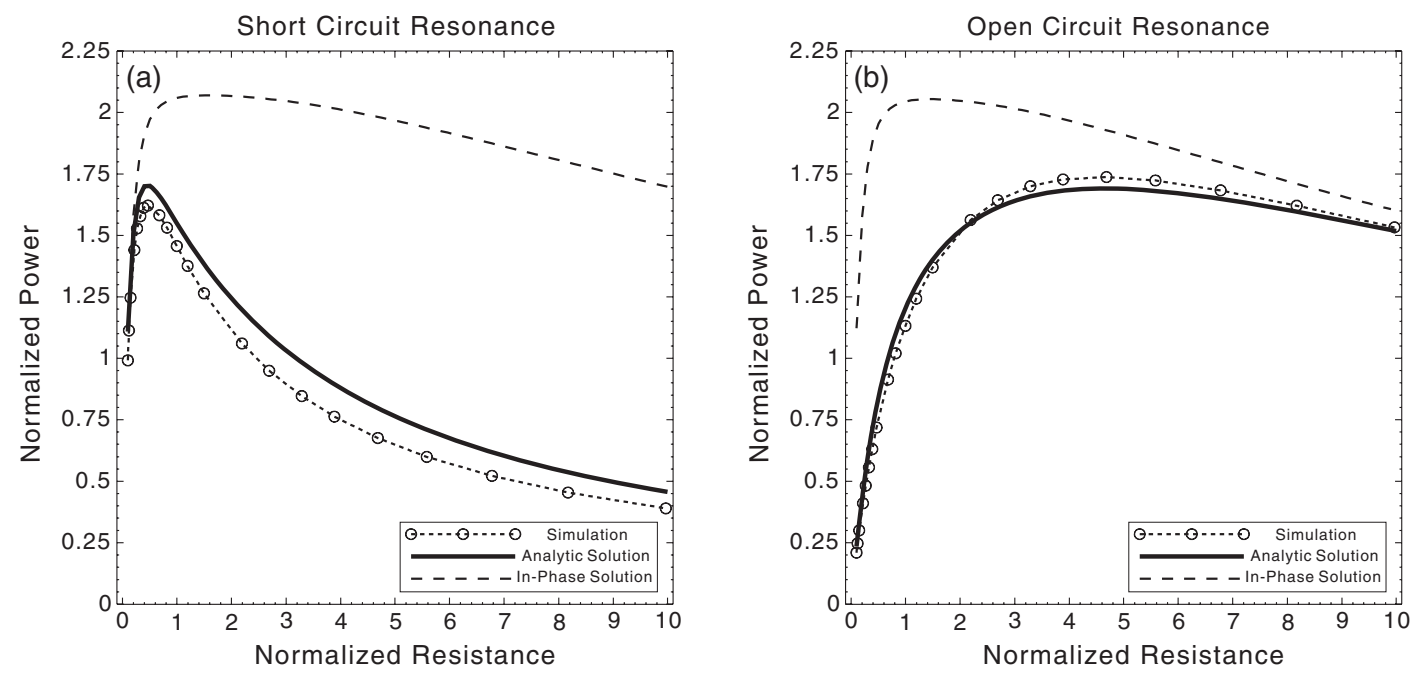

Figure 7. Numerical validation for medium $\frac{k_{e}^{2}}{\zeta}$. We use $k_{e}=0.4$ and $\zeta=0.03\left(\frac{k_{e}^{2}}{\zeta}=5.33\right)$. The normalized power versus normalized resistance is plotted in (a) operated at $\Omega_{\mathrm{sc}}$ and in (b) operated at $\Omega_{\mathrm{oc}}$.
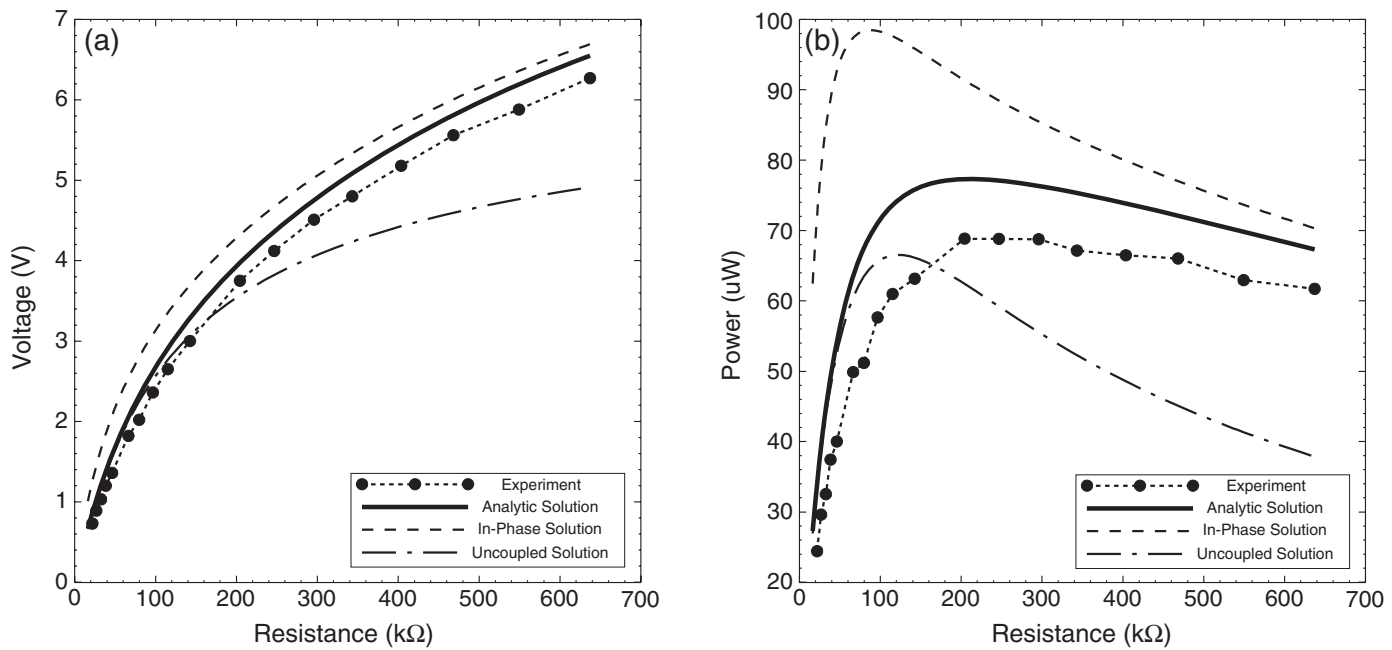

Figure 8. Experimental validation for medium $\frac{k_{e}^{2}}{\zeta}$. The structure is excited at its open circuit resonance. The parameters $k_{e}$ and $\zeta$ are measured to be 0.17 and $0.01\left(\frac{k_{e}^{2}}{\zeta}=2.89\right)$. (a) Rectified voltage versus resistance. (b) Harvested power versus resistance.

not realistic if $\frac{k_{e}^{2}}{\zeta}$ is not small. We then omit them here. The normalized displacement, voltage and power are plotted against the normalized electric resistance and frequency in figures 9(a)-(c) calculated based on the analytic solutions as well as in figures 9(d)-(f) calculated based on the in-phase solutions. In figure 9(c) we see clearly that the harvested power has two optimal values of equal amount evaluated at two different resistances and frequencies; i.e. $\bar{P}$ is maximized at

$$
\begin{aligned}
\left(r_{1}^{\mathrm{opt}}, \Omega_{1}^{\mathrm{opt}}\right) & =(0.062,1.025), \\
\left(r_{2}^{\mathrm{opt}}, \Omega_{2}^{\mathrm{opt}}\right) & =(17.299,1.492) .
\end{aligned}
$$

Note that (60) confirms our theoretical predictions made in section 3.3 which shows that $r_{1}^{\mathrm{opt}} \ll 1$ and $\Omega_{1}^{\mathrm{opt}} \approx \Omega_{\mathrm{sc}}$ and $r_{2}^{\text {opt }} \gg 1$ and $\Omega_{2}^{\text {opt }} \approx \Omega_{\mathrm{oc}}$. However, the in-phase approach fails to predict the optimal operating frequencies since there are always two optimal electric loads for each applied frequency as shown in figure 9(f). The effect of the optimal selection of operating frequency in generating the desired properties is neglected in the in-phase analysis, which turns out to be important in the design criterion.

Switching between these two peaks can be achieved by varying the electric loads along the curve $r^{\text {opt }}\left(\Omega, k_{e}, \zeta\right)$ obtained from (31). The implication of this result can be applied to enhancing the efficiency of charging a battery. Indeed, Ottman et al [28] have shown that the efficiency of direct charging of a battery without a suitable controller is pretty slow. The main reason for this is that the equivalent electrical resistance of a battery is much smaller than the optimal electrical resistance. Turning the load impedance needs a special power converter [29], which in turn may consume additional extracted energy and make the circuitry unrealistic (see the discussion of [43]). Alternatively, if the piezoelectric generator has a pronounced frequency shift and 

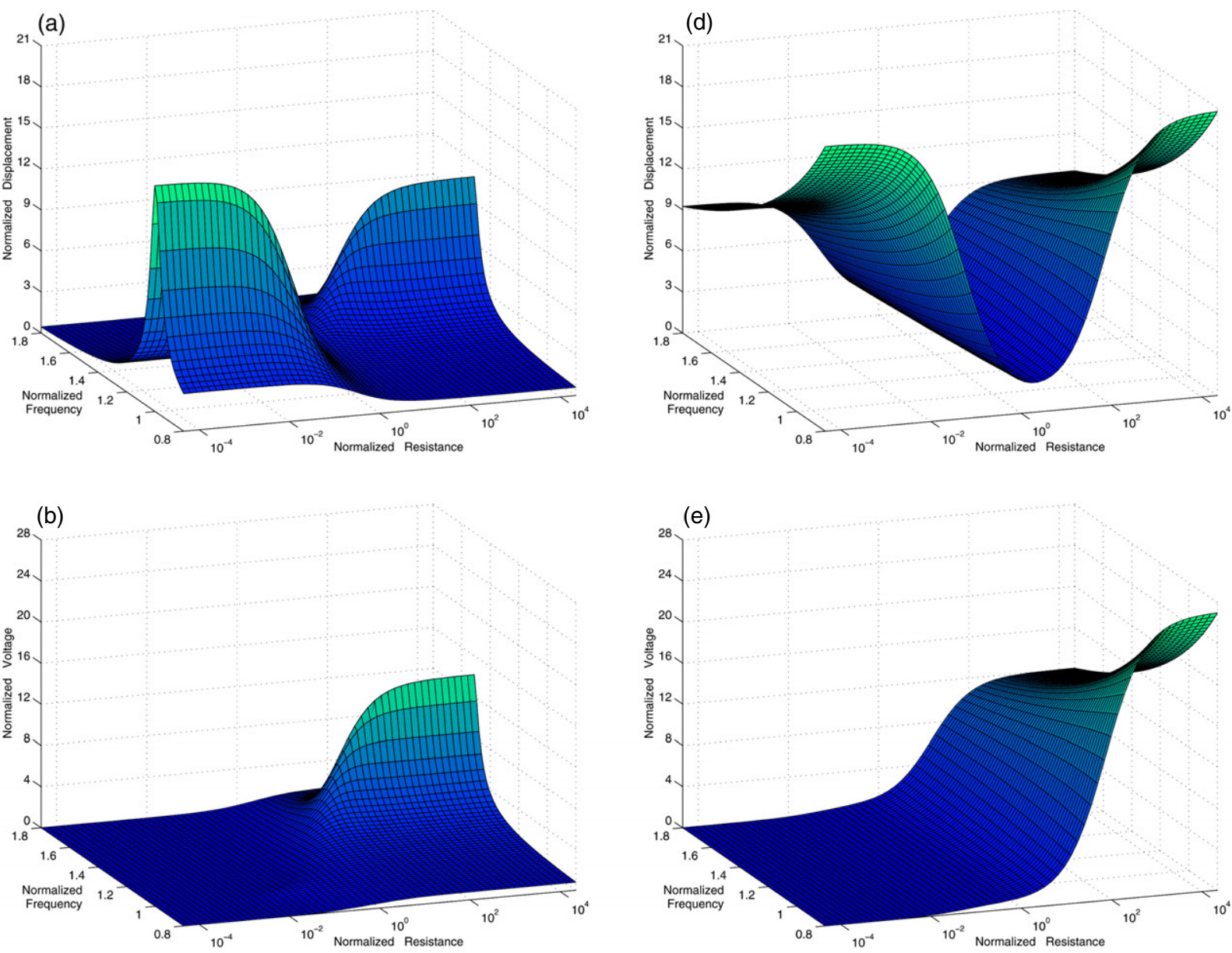

(c)

(f)
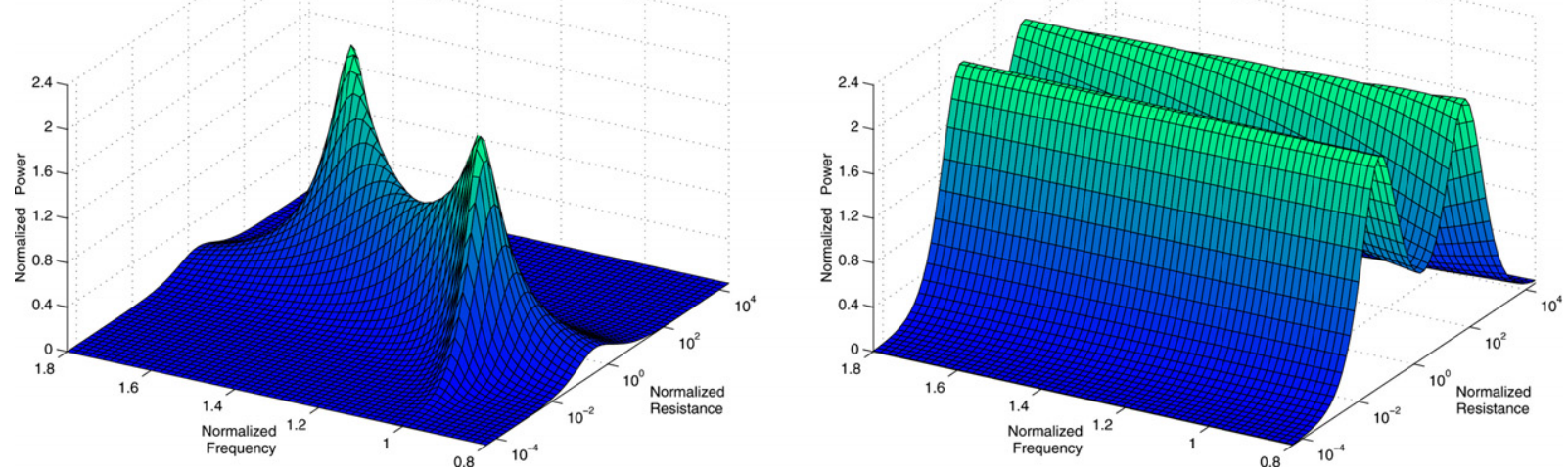

Figure 9. Comparisons for large $\frac{k_{e}^{2}}{\zeta}$. We use $k_{e}=1.14$ and $\zeta=0.03\left(\frac{k_{e}^{2}}{\zeta}=43.3\right)$. The normalized displacement, voltage and power are plotted against the normalized electric resistance and frequency in (a)-(c) calculated based on the analytic solutions as well as in (d)-(f) calculated based on the in-phase solutions.

(This figure is in colour only in the electronic version)

large $\frac{k_{e}^{2}}{\zeta}$, the equivalent impedance of a battery can be matched to the optimal load by selecting a suitable operating point close to $\Omega_{\mathrm{sc}}$ since the harvested power has a peak around there.

Next, unlike the power, the displacement and voltage evaluated at these two optimal conditions (60) differ significantly (compare figures 9 (a) and (b) with (c)). The displacement has two hills with one chain concentrated at $\Omega_{\mathrm{sc}}$ and the other at $\Omega_{\mathrm{oc}}$. But unlike the power, it is larger at $\Omega_{\mathrm{sc}}$ than at $\Omega_{\mathrm{oc}}$ since the overall damping of system is higher at the open circuit resonance (see also the predictions in table 1). The advantage of operating at the second peak close to $\Omega_{\mathrm{oc}}$ is space-saving if the smaller device is preferred.

Finally, figure 9(b) clearly demonstrates that the normalized rectified voltage evaluated at $\left(r_{2}^{\text {opt }}, \Omega_{2}^{\text {opt }}\right)$ is one order of magnitude higher than that evaluated at $\left(r_{1}^{\mathrm{opt}}, \Omega_{1}^{\mathrm{opt}}\right)$. It is thus advantageous to operate at the open circuit resonance to 
overcome the minimum voltage requirement of the rectifying bridge in the micro-scale device. On the other hand, the steady-state current evaluated at the first peak of power around $\Omega_{\mathrm{sc}}$ is one order of magnitude higher than that evaluated at the second peak of power around $\Omega_{\mathrm{oc}}$, since $P=I V$ and the optimal power is identical at these two peaks. We may apply this result to charging batteries. Indeed, optimizing the power flowing into the battery is equivalent to maximizing the current into it as the battery voltage is essentially constant or only changes slowly. Hence, operating at the short circuit resonance together with the corresponding optimal load could enhance the efficiency of charging the battery directly without adjustable convectors.

\section{Conclusions}

We study the optimal AC-DC power output for a vibrating piezoelectric generator connected to an energy harvesting circuit. In contrast with estimates proposed by the uncoupled and in-phase approaches $[10,28]$, we show that the power extraction depends on the input vibration characteristics (frequency and acceleration), the mass of the generator, the electrical load, the natural frequency, the mechanical damping ratio, and the electromechanical coupling coefficient of the system. An expression for average harvested power that incorporates all of these factors is analytically developed by (30). Thus, the scheme to optimize the power either by tuning the electric resistance, selecting suitable operation points, or adjusting the system coupling coefficient by optimal structural design can be guided completely by (30). Further, it is also highly recommended that all these parameters be provided in all future publications to facilitate the relative comparison of various devices.

We compare our approach to others proposed based on the uncoupled and in-phase assumptions. We show that the conventional uncoupled solution is suitable provided that the ratio $\frac{k_{e}^{2}}{\zeta} \ll 1$, while the discrepancies between these distinct approaches become significant when $\frac{k_{e}^{2}}{\zeta}$ increases. Figures 6 and 9 highlight the striking contrast in normalized displacement, voltage and power output calculated based on the uncoupled, in-phase and analytic solutions for non-small ratio $\frac{k_{e}^{2}}{\zeta}$. We perform a series of experiments and numerical simulations to evaluate these approaches. We find our analytic solutions are in good agreement with both experiments and simulations as shown in figures 7 and 8 . The in-phase solutions, however, overestimate the measured voltage and power and underestimate the optimal load compared with experimental observations.

We make a particular study of the important case when the shift in device natural frequency is pronounced and the quality factor of the system is large, since this has been neglected by most current optimization schemes, as pointed out by [5]. The effect of this frequency shift is expected to be more pronounced for micro-scale harvesters, since the contribution of piezoelectric elements to the overall structural stiffness is much larger than for bulk generators. In this situation, the harvested power is shown to have two optima evaluated at $\left(r_{1}^{\mathrm{opt}}, \Omega_{1}^{\mathrm{opt}}\right)$ and $\left(r_{2}^{\mathrm{opt}}, \Omega_{2}^{\mathrm{opt}}\right)$, where $\Omega_{1}^{\mathrm{opt}}$ is close to $\Omega_{\mathrm{sc}}$ and the electric load $r_{1}^{\text {opt }}$ is very small, while $\Omega_{2}^{\text {opt }}$ is close to $\Omega_{\mathrm{oc}}$ and $r_{2}^{\text {opt }}$ is large. Table 1 sheds light on the conspicuous contrast in the normalized displacement, electric resistance, voltage, current and power evaluated at these two power optimal pairs. Finally, for devices with strong electromechanical coupling, several design guidelines including enhancing the efficiency of charging a battery directly are recommended.

\section{Acknowledgments}

We thank Professors K C Wu and C K Lee for their constant encouragement and support. We are grateful to Professors C S Yeh, K C Chen, W J Wu and W P Shih for many helpful and stimulating discussions. We are glad to acknowledge the Ministry of Economic Affair for support under grant no 94-EC17-A-05-S1-017 (WHAM-BioS).

\section{References}

[1] Allen J J and Smits A J 2001 Energy harvesting EEL J. Fluids Struct. 15 629-40

[2] Badel A, Guyomar D, Lefeuvre E and Richard C 2005 Efficiency enhancement of a piezoelectric energy harvesting device in pulsed operation by synchronous charge inversion J. Intell. Mater. Syst. Struct. 16 889-901

[3] Chandrakasan A, Amirtharajah R, Goodman J and Rabiner W 1998 Trends in low power digital signal processing Int. Symp. Circuits Syst. 4 604-7

[4] Cornwell P J, Goethal J, Kowko J and Damianakis M 2005 Enhancing power harvesting using a tuned auxiliary structure J. Intell. Mater. Syst. Struct. 16 825-34

[5] duToit N E, Wardle B L and Kim S G 2005 Design considerations for MEMS-scale piezoelectric mechanical vibration energy harvesters Integr. Ferroelectr. 71 121-60

[6] Elvin N, Elvin A and Choi D H 2003 A self-powered damage detection sensor J. Strain Anal. 38 115-24

[7] Elvin N G, Elvin A A and Spector M 2001 A self-powered mechanical strain energy sensor Smart Mater. Struct. 10 293-9

[8] Ericka M, Vasic D, Costa F, Poulin G and Tliba S 2005 Energy harvesting from vibration using a piezoelectric membrane J. Physique Coll. 128 187-93

[9] Goldfarb M and Jones L D 1999 On the efficiency of electric power generation with piezoelectric ceramic Trans. ASME, J. Dyn. Syst., Meas. Control 121 566-71

[10] Guyomar D, Badel A and Lefeuvre E 2005 Toward energy harvesting using active materials and conversion improvement by nonlinear processing IEEE Trans. Ultrason. Ferroelectr. Freq. Control $\mathbf{5 2} 584-95$

[11] Hagood N W, Chung W H and Flotow A V 1990 Modelling of piezoelectric actuator dynamics for active structural control J. Intell. Mater. Syst. Struct. 1327-54

[12] Hong Y K, Park H K, Lee S Q, Moon K S, Vanga R R and Levy M 2004 Design and performance of a self-sensing, self-actuating piezoelectric monomorph with interdigitated electrodes Proc. SPIE Int. Conf. on Optomechatronic Sensors, Actuators, and Control (Philadelphia, Oct. 2004) vol 5602, pp 210-7

[13] Jeon Y B, Sood R, Jeong J H and Kim S G 2005 MEMS power generator with transverse mode thin film PZT Sensors Actuators A 122 16-22

[14] Kim H W, Batra A, Priya S, Uchino K, Markley D, Newnham R E and Hofmann H F 2004 Energy harvesting using a piezoelectric cymbal transducer in dynamic environment Japan. J. Appl. Phys. 43 6178-83

[15] Kim H W, Priya S, Uchino K and Newnham R E 2005 Piezoelectric energy harvesting under high pre-stressed cyclic vibrations J. Electroceram. 15 27-34 
[16] Kim S, Clark W W and Wang Q M 2005 Piezoelectric energy harvesting with a clamped circular plate: analysis J. Intell. Mater. Syst. Struct. 16 847-54

[17] Kim S, Clark W W and Wang Q M 2005 Piezoelectric energy harvesting with a clamped circular plate: experimental study J. Intell. Mater. Syst. Struct. 16 855-63

[18] Lefeuvre E, Badel A, Benayad A, Lebrun L, Richard C and Guyomar D 2005 A comparison between several approaches of piezoelectric energy harvesting J. Physique Coll. 128 $177-86$

[19] Lefeuvre E, Badel A, Richard C and Guyomar D 2005 Piezoelectric energy harvesting device optimization by synchronous electric charge extraction J. Intell. Mater. Syst. Struct. 16 865-76

[20] Lesieutre G A 1998 Vibration damping and control using shunted piezoelectric materials Shock Vib. Digest 30 187-95

[21] Lesieutre G A and Davis C L 1997 Can a coupling coefficient of a piezoelectric device be higher than those of its active material? J. Intell. Mater. Syst. Struct. 8 859-67

[22] Lesieutre G A, Ottman G K and Hofmann H F 2004 Damping as a result of piezoelectric energy harvesting $J$. Sound Vib. 269 991-1001

[23] Liao W H, Wang D H and Huang S L 2001 Wireless monitoring of cable tension of cable-stayed bridges using PVDF piezoelectric films J. Intell. Mater. Syst. Struct. 12 331-9

[24] Lu F, Lee H P and Lim S P 2004 Modeling and analysis of micro piezoelectric power generators for micro-electro-mechanical-systems applications Smart Mater Struct. 13 57-63

[25] Mateu L and Moll F 2005 Optimum piezoelectric bending beam structures for energy harvesting using shoe inserts J. Intell. Mater. Syst. Struct. 16 835-45

[26] Mossi K, Green C, Ounaies Z and Hughes E 2005 Harvesting energy using a thin unimorph prestressed bender: geometrical effects J. Intell. Mater. Syst. Struct. 16 249-61

[27] Ng T H and Liao W H 2005 Sensitivity analysis and energy harvesting for a self-powered piezoelectric sensor J. Intell. Mater. Syst. Struct. 16 785-97

[28] Ottman G K, Hofmann H F, Bhatt A C and Lesieutre G A 2002 Adaptive piezoelectric energy harvesting circuit for wireless remote power supply IEEE Trans. Power Electron. 17 669-76

[29] Ottman G K, Hofmann H F and Lesieutre G A 2003 Optimized piezoelectric energy harvesting circuit using step-down converter in discontinuous conduction mode IEEE Trans. Power Electron. 18 696-703

[30] Poulin G, Sarraute E and Costa F 2004 Generation of electric energy for portable devices: comparative study of an electromagnetic and a piezoelectric system Sensors Actuators A 116 461-71

[31] Priya S, Chen C T, Fye D and Zahnd J 2005 Piezoelectric windmill: a novel solution to remote sensing Japan. J. Appl. Phys. 44 L104-7

[32] Rabaey J M, Ammer M J, da Silva J L Jr, Patel D and Roundy S 2000 Picoradio supports ad hoc ultra-low power wireless networking Computer 33 42-8

[33] Ramsay M J and Clark W W 2001 Piezoelectric energy harvesting for bio MEMS applications Proc. SPIE 4332 429-38
[34] Richards C D, Anderson M J, Bahr D F and Richards R F 2004 Efficiency of energy conversion for devices containing a piezoelectric component J. Micromech. Microeng. $14717-21$

[35] Roundy S 2005 On the effectiveness of vibration-based energy harvesting J. Intell. Mater. Syst. Struct. 16 809-23

[36] Roundy S, Leland E S, Baker J, Carleton E, Reilly E, Lai E, Otis B, Rabaey J M, Wright P K and Sundararajan V 2005 Improving power output for vibration-based energy scavengers IEEE Pervasive Comput. 4 28-36

[37] Roundy S, Steingart D, Frechette L, Wright P and Rabaey J 2004 Power sources for wireless sensor networks Lect. Notes Comput. Sci. 2920 1-17

[38] Roundy S and Wright P K 2004 A piezoelectric vibration based generator for wireless electronics Smart Mater. Struct. $131131-42$

[39] Roundy S, Wright P K and Rabaey J 2003 A study of low level vibrations as a power source for wireless sensor nodes Comput. Commun. 26 1131-44

[40] Shenck N S and Paradiso J A 2001 Energy scavenging with shoe-mounted piezoelectrics IEEE Micro 21 30-42

[41] Sodano H A, Inman D J and Park G 2004 A review of power harvesting from vibration using piezoelectric materials Shock Vib. Digest 36 197-205

[42] Sodano H A, Inman D J and Park G 2005 Comparison of piezoelectric energy harvesting devices for recharging batteries J. Intell. Mater. Syst. Struct. 16 799-807

[43] Sodano H A, Inman D J and Park G 2005 Generation and storage of electricity from power harvesting devices J. Intell. Mater. Syst. Struct. 16 67-75

[44] Sodano H A, Park G and Inman D J 2004 Estimation of electric charge output for piezoelectric energy harvesting J. Strain $4049-58$

[45] Starner T 1996 Human-powered wearable computing IBM Syst. J. 35 618-29

[46] Taylor G W, Burns J R, Kammann S M, Powers W B and Welsh T R 2001 The energy harvesting eel: a small subsurface ocean/river power generator IEEE J. Ocean. Eng. 26 539-47

[47] Trolier-Mckinstry S and Muralt P 2004 Thin film piezoelectrics for MEMS J. Electroceram. 12 7-17

[48] Umeda M, Nakamura K and Ueha S 1996 Analysis of the transformation of mechanical impact energy to electric energy using piezoelectric vibrator Japan. J. Appl. Phys. $353267-73$

[49] Umeda M, Nakamura K and Ueha S 1997 Energy storage characteristics of a piezo-generator using impact induced vibration Japan. J. Appl. Phys. 36 3146-51

[50] Wang Q M and Cross L E 1999 Constitutive equations of symmetrical triple layer piezoelectric benders IEEE Trans. Ultrason. Ferroelectr. Freq. Control 46 1343-51

[51] White N M, Glynne-Jones P and Beeby S P 2001 A novel thick-film piezoelectric micro-generator Smart Mater. Struct. 10 850-2

[52] Williams C B and Yates R B 1996 Analysis of a micro-electric generator for microsystems Sensors Actuators A 52 8-11

[53] Yoon H S, Washington G and Danak A 2005 Modeling, optimization, and design of efficient initially curved piezoceramic unimorphs for energy harvesting applications J. Intell. Mater. Syst. Struct. 16 877-88 